\title{
Synthesis and Statistical Optimization of Poly (Lactic-Co-Glycolic Acid) Nanoparticles Encapsulating GLPI Analog Designed for Oral Delivery
}

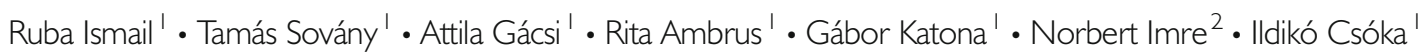

Received: 17 January 2019 / Accepted: 1 April 2019 / Published online: 13 May 2019

(C) The Author(s) 2019

\begin{abstract}
Purpose To design and stabilize Liraglutide loaded poly (lactic-co-glycolic acid) nanoparticles (PLGA NPs) proper for oral administration.

Methods PLGA NPs were prepared by means of double emulsion solvent evaporation method and optimized by applying 7-factor 2-level Plackett-Burman screening design.

Results Spherical shaped NPs with homogeneous distribution, $188.95 \mathrm{~nm}$ particle size and $51.81 \%$ encapsulation efficiency were obtained. Liraglutide was successfully entrapped in the NPs while maintaining its native amorphous nature, and its structural integrity as well.

Conclusion Lira-PLGA NPs with the required Critical Quality Attributes (CQAs) were successfully designed by implementing a 7-factor 8-run Plackett Burman design into the extended Quality by Design ( $\mathrm{QbD}$ ) model, to elucidate the effect of formulation and process variables on the particle size, size-distribution, encapsulation efficiency and surface charge. As the developed nanoparticles maintained the native structure of the active pharmaceutical ingredient (API), they are promising compositions for the further development for the oral delivery of Lira.
\end{abstract}

KEY WORDS liraglutide · oral delivery · plackett Burman design · PLGA nanoparticles $\cdot$ quality by design

Electronic supplementary material The online version of this article (https://doi.org/l 0. 1007/s I 1095-0 19-2620-9) contains supplementary material, which is available to authorized users.

Ildikó Csóka

csoka@pharm.u-szeged.hu

University of Szeged, Faculty of Pharmacy, Institute of Pharmaceutical Technology and Regulatory Affairs, Eötvös u. 6, Szeged H-6720, Hungary

2 University of Szeged, Faculty of Pharmacy, Institute of Pharmaceutical Analysis, Somogyi u. 4, Szeged H-6720, Hungary

\section{INTRODUCTION}

A current scenario in pharmaceutical development is inclined towards employing rational Quality by Design $(\mathrm{QbD})$ strategy $(1,2)$ which has been adopted by the pharmaceutical industry to guarantee the quality of drug products (3). One of the key elements of $\mathrm{QbD}$ is to identify and thoroughly understand formulation and process variables and their effects on the critical quality attributes (CQAs), followed by the optimization of these variables by applying an appropriate statistical design of experiment (DOE) which enables the researcher to minimize the number of runs and helps in identifying the most influential parameters namely; critical process parameters (CPPs) and critical material attributes (CMAs) which may highly impact the quality of the product. In addition to that, DOE helps in identifying the optimum level of each factor that assures the desired CQAs values, to comply with the desired Quality Target Product Profile (QTPP) (4). One of the important questions when implementing DoE methodology is the selection of adequate experimental design.that matches the experimental objective. When estimating the main effects of large number of factors are of interest to be investigated, screening designs such as 2- level Plackett-Burman (PB) is applied. The main advantage of applying such screening designs is the minimum number of observations needed to calculate the effect of several variables. If providing further information on direct and pairwise-interaction effects and curvilinear variable effects is desired, second order designs: central composite designs (CCD) and Box Behncken designs (BBD) are the most widely applied ones $(5,6)$. CCD provides better prediction capability than $\mathrm{BBD}$, while the latter requires fewer runs in case of 3 or 4 variables and is applied when combined factor extremes should be avoided.

Liraglutide (Lira) or NN2211 is a recombinant palmitylacylated derivative of glucagon like peptide -1 (GLP1), which 
was approved for the treatment of T2DM (6) in addition to chronic weight management (7). Lira is currently administered once daily through subcutaneous injection which is an invasive route known to be limited by insufficient patient adherence to the therapy in addition to the fact that this therapeutic route is not strictly mimicking the physiological secretion route of GLP1 (8). The oral route should be regarded as the most desirable choice for the delivery of Lira (9), and realizing the dream of administering a GLP1 analog such as Lira orally is still an elusive goal despite all advances in peptide delivery systems.

Based on our careful evaluation of literature regarding the emerging developments in oral delivery of antidiabetic peptides (10,11), we found out that PLGA nanoparticles showed promising results in improving the stability of peptides through the GIT in addition to other merits of nanocarrier systems, which can all lead to enhancing the oral bioavailability of these peptides (12). Among the applied techniques for preparing PLGA NPs, the double emulsion solvent evaporation method was found to be the most preferable one, which has been efficiently used for encapsulating peptides and proteins (13-15). Nevertheless, the physicochemical properties of nanoparticles may be affected by various formulation and process parameters, which influence the product quality.

To the best of our knowledge, there is no previously reported work considering the application of DOE as a part of the QbD strategy for the development of PLGA NPs encapsulating GLP-1 analog. Here we focus on the optimization of the size and EE of the NPs as a crucial demand along with the polydisperisity index (PDI) and surface charge. The design space (DS) was established to optimize the level of each of the examined factors, then surface morphology, compatibility studies as well as structural and conformational stability tests of Lira encapsulated in the optimized formula were conducted on the optimized formula.

\section{MATERIALS AND METHODS}

\section{Materials}

Liraglutide was purchased from Xi'an Health Biochem Technology Co., Ltd. (China), Poly(lactide-co-glycolide) (PLGA 50:50, Mw = 30,000-60,000 Da), PVA (MOWIOL 4-98, MW 27,000 Da) which is a soluble polymer, and D- $(+)$-Trehalose dihydrate $(\mathrm{MW}=378.33 \mathrm{~g} / \mathrm{mol}$ ) were purchased from Sigma Aldrich (Germany). D-(-)Mannitol was purchased from Molar Chemicals Ltd. (Hungary). Sodium acetate anhydrous was purchased from Scharlau Chemie S.A. (Spain). Ethyl acetate used for dissoloving PLGA was from REANAL Labor
(Hungary). All other chemicals in the study were of analytical reagent grade.

\section{Methods}

\section{Preparation of Liraglutide Loaded PLGA NPs Using Double Emulsion Solvent Evaporation Method}

The preparation of Liraglutide loaded PLGA nanoparticles was carried out by means of the double emulsion W1/O/W2solvent evaporation method, which is the most commonly used technique for the encapsulation of peptide drugs within PLGA NPs due to its simplicity and high encapsulation efficiency $(15,16)$. The amount of PLGA (30 or $60 \mathrm{mg}$ ) was dissolved in ethyl acetate at room temperature to form the organic phase. Ethyl acetate was the organic solvent of choice here as it was reported to increase the rate of encapsulation of hydrophilic molecules (16). The inner aqueous phase of $0.5-$ $5 \mathrm{mg}$ liraglutide was dissolved in $0.5 \mathrm{ml}$ of $1 \%$ sodium acetate aqueous solution, it was slowly added to the organic phase, then water/oil primary emulsion was formed upon sonication at the power of $90 \mathrm{~W}$ for $30 \mathrm{~s}$ using a probe sonicator in ice bath. The obtained emulsion was re-emulsified with external aqueous phase containing $0.5-2 \%$ PVA as stabilizer by sonication in ice bath at the power of $90 \mathrm{~W}$ for $0.5-2 \mathrm{~min}$ using the probe sonicator. The obtained water-in-oil-in-water $(\mathrm{W} 1 / \mathrm{O} /$ W2) double emulsion was subjected to magnetic stirring at room temperature over the night to allow the complete evaporation of ethyl acetate. The nanoparticles were then collected by centrifugation for $15 \mathrm{~min}$ at $16500 \mathrm{rpm}$, washed three times with distilled water, and resuspended in deionized water.

For the lyophilization process, $1.5 \mathrm{ml}$ of each nanoparticles suspension was poured into semi-stoppered glass vials with slotted rubber closures and freeze-dried at $-40^{\circ} \mathrm{C}$ for 72 h. 5-10\% mannitol or trehalose was added as lyoprotectants. The chamber pressure was maintained at 0.01 mbar, and the process was controlled by (Scanlaf CTS16a02) software.

\section{Design of Experiment Study Using Plackett Burman Design}

PB design is the most widely used method among the various screening designs used for the determination of the most influential factors affecting the pharmaceutical development as it has many advantages: it screens a large number of variables and identifies the highly influential ones with relatively few runs, while assuring a good degree of accuracy. PB design with a total of 8 runs involving 7 independent variables was carried out using STATISTICA 13 software, and analysis of variance (ANOVA) was applied to determine the statistical significance of each model coefficient, which was significant at 95\% level $(P<0.05)$. 
The linear equation of this model is:

$$
\begin{aligned}
\mathrm{Y}= & \mathrm{b}_{0}+\mathrm{b}_{1} \mathrm{X}_{1}+\mathrm{b}_{2} \mathrm{X}_{2}+\mathrm{b}_{3} \mathrm{X}_{3}+\mathrm{b}_{4} \mathrm{X}_{4}+\mathrm{b}_{5} \mathrm{X}_{5}+\mathrm{b}_{6} \mathrm{X}_{6} \\
& +\mathrm{b}_{7} \mathrm{X}_{7}
\end{aligned}
$$

where $\mathrm{Y}$ is the response, $\mathrm{b}_{0}$ is the constant and $\mathrm{b}_{1}, \mathrm{~b}_{2} \ldots \mathrm{b}_{7}$ are the coefficient of factors $\mathrm{X}_{1}, \mathrm{X}_{2} \ldots \mathrm{X}_{7}$. It is known that a positive value of the regression coefficient is an indicator of a positive effect of the factor $(\mathrm{X})$ on the response $(\mathrm{Y})$, while a negative value refers to an inverse relation between the examined variable and the response (17).

Depending on our previous risk assessment-based investigation (18-20), the selected independent formulation and process variables were: PLGA amount (X1), liraglutide amount (X2), 2nd sonication time (X3), PVA concentration (X4), lyoprotectant type (X5), lyoprotectant concentration (X6) and external aqueous phase to organic phase $\mathrm{w} 2 / \mathrm{o}$ ratio (X7). Particle size (Y1), PDI (Y2), EE (Y3) and zeta potential (Y4) were selected as dependent variables. The examined lower and upper levels of the independent factors $\mathrm{X}_{1}-\mathrm{X}_{7}$ (Table 1) were also determined depending on preliminary experiments and literature survey.. The design was validated by 3 extra center checkpoint formulations and the bias $(\%)$ between predicted and observed values of response was calculated. The optimized formulation was prepared within design space (DS) and compared with predicted results of the responses.

\section{Characterization of the Prepared Liraglutide Loaded PLGA NPS}

Particle Size, Size Distribution and Surface Charge Measurements. Approximately $5 \mathrm{mg}$ of the prepared freezedried NPs was dispersed in $5 \mathrm{ml}$ of double distilled water and sonicated to minimize the possible inter-particle interactions. The hydrodynamic diameter (Z-average), PDI and zeta potential of reconstituted NPs were measured in folded capillary cell by using Malvern Nano ZS Zetasizer (Malvern Instruments Ltd. UK) equipped with He-Ne laser(633 nm). The instrument allows the particle size measurement in the range of $0.3 \mathrm{~nm}-10.0 \mu \mathrm{m}$ using patented NIBS (Non-Invasive Back Scatter) technology, with high accuracy of $\pm 2 \%$. The samples were measured at $25^{\circ} \mathrm{C}$, the refractive index was 1.445 , and the number of scans was 17. All the measurements were conducted in triplicate and the average value of each was used.

Encapsulation Efficiency (EE). The encapsulation efficiency of liraglutide encapsulated in PLGA NPs was determined directly using the centrifugation method. In this method, $20 \mathrm{mg}$ from each NPs formulation was dissolved in $2 \mathrm{ml}$ of DCM, then liraglutide was extracted into $4 \mathrm{ml}$ of PBS $(\mathrm{pH}=8.1)$, soaked for $30 \mathrm{~min}$ and then centrifuged at $16500 \mathrm{rpm}$ at

\begin{tabular}{|c|c|c|c|}
\hline \multicolumn{2}{|l|}{ Critical factor } & \multirow{2}{*}{$\begin{array}{l}\text { Low level } \\
30\end{array}$} & \multirow{2}{*}{$\begin{array}{l}\text { High leve } \\
60\end{array}$} \\
\hline PLGA amount (mg) & $X I$ & & \\
\hline Liraglutide amount (mg) & $\times 2$ & 0.5 & 5 \\
\hline 2nd sonication time ( $\mathrm{min}$ ) & $\times 3$ & 0.5 & 2 \\
\hline PVA (\%) & $\times 4$ & 0.5 & 2 \\
\hline Lyoprotectant type & $\times 5$ & Mannitol & Trehalose \\
\hline Lyoprotectant (\%) & $\times 6$ & 5 & 10 \\
\hline W2/O ratio & $\times 7$ & 2 & 5 \\
\hline
\end{tabular}

Table I Levels of the Selected Critical Factors

$4^{\circ} \mathrm{C}$ for $15 \mathrm{~min}$. The supernatant was then collected and the amount of encapsulated liraglutide in the supernatant was measured using the RP-HPLC method. Samples were run in triplicate.

The percentage of EE was calculated using the following equations:

$\mathrm{EE} \%=$ encapsulated amount of liraglutide/ total amount of liraglutide added* 100.

Chromatographic Equipment and Conditions. Reversed phase HPLC (Shimadzu Corporation, NEXERA X2, Tokyo, Japan) method was developed in our lab to analyze liraglutide. A Kinetex ${ }^{\circledR}$ C 18 column with dimensions of $(5 \mu \mathrm{m}$, $150 * 4.6 \mathrm{~mm}$, (Phenomenex, USA) was used as a stationary phase. The flow rate of $1.5 \mathrm{ml} / \mathrm{min}$ was set over $15 \mathrm{~min}$ with a mobile phase comprised of $0.02 \mathrm{M}$ aqueous KH2PO4 solution ( $\mathrm{pH}=7.0$, solvent $\mathrm{A}$ ) and acetonitrile (solvent $\mathrm{B}$ ). The mobile phase was pumped in a gradient mode as it was changed from 80:20 (A:B, v/v) to $30: 70(\mathrm{~A}: \mathrm{B}, \mathrm{v} / \mathrm{v})$ in $12 \mathrm{~min}$ then going back to $80: 20(\mathrm{v} / \mathrm{v})$ between $12.1-15 \mathrm{~min}$. The column temperature was set to $40^{\circ} \mathrm{C}$, and the sample tray temperature was set to $15^{\circ} \mathrm{C}$. Fifty microliters of sample volume was injected. The wavelength of UV detection was $214 \mathrm{~nm}$.. The retention time of liraglutide is $8.65 \mathrm{~min}$. In our HPLC method, some chromatographic parameters have been calculated. The limit of detection (LOD) value of the liraglutide is $0.175 \mathrm{ppm}$, the limit of quantification (LOQ) value is $0,530 \mathrm{ppm}$, respectively. Capacity factor ( $\left.k^{\prime}\right)$ for liraglutide is 5.20, the asymmetry factor of the peak of liraglutide showed 1.40 value, respectively. The theoretical plate $(\mathrm{N})$ value is 146,620 , calculated by the Ph.Eur. guideline. The regression of the linearity $\left(\mathrm{R}^{2}\right)$ of the liraglutide calibration curve was 0.996 , respectively.

Scanning Electron Microscopy Measurements (SEM). To investigate the surface morphology, sphericity, and discreteness of the freeze dried NPs containing lira, scanning electron 
microscopy (SEM) (Hitachi S4700, Hitachi Scientific Ltd., Tokyo, Japan) at $10 \mathrm{kV}$ was used. The samples were coated with gold-palladium (90 s) with a sputter coater (Bio-Rad SC 502, VG Microtech, Uckfield, UK) using an electric potential of $2.0 \mathrm{kV}$ at $10 \mathrm{~mA}$ for $10 \mathrm{~min}$. The air pressure was $1.3-$ $13.0 \mathrm{mPa} .3$ repetitions of the optimized formula were tested by SEM technique and images were captured from different surface regions of each sample and at two different magnifications $(\times 15,000, \times 45,000)$.

\section{Compatibility Studies}

To investigate the physicochemical compatibility between the drug and the polymer in the prepared PLGA NPs, FTIR, DSC and XRD analysis were conducted.

Fourier Transform Infrared Spectroscopy (FTIR). The FT-IR spectra of pure Lira, PLGA, Lira free/loaded PLGA NPs were recorded using FT-IR spectrometer (Thermo Nicolet AVATAR; LabX Midland, ON, Canada) in the range of 4000 and $400 \mathrm{~cm}^{-1}$ with an optical resolution of $4 \mathrm{~cm}^{-1}$. The sample was mixed with $150 \mathrm{mg}$ of dry $\mathrm{KBr}$ and compressed to prepare the pellet.

Differential Scanning Calorimetry (DSC). To define the physical state of the peptide drug in the nanoparticles and assess any possible intermolecular interaction between the drug and the polymer in the nanoparticles, DSC studies of pure Lira, PLGA, Lira free/loaded PLGA NPs were performed using (Mettler Toledo TG 821e DSC Mettler Inc., Schwerzenbach, Switzerland). Accurately weighed samples $(3-5 \mathrm{mg}$ ) were sealed in an aluminum pan and an empty pan was used as a reference. The samples were analyzed at a scanning temperature from 25 to $300^{\circ} \mathrm{C}$ at a heating rate of $10^{\circ} \mathrm{C} / \mathrm{min}$ under nitrogen purge. Data analysis was performed using the $\mathrm{STAR}^{\mathrm{e}}$ software (Mettler Toledo Mettler Inc., Schwerzenbach, Switzerland).

$X$-Ray Diffraction Study (XRD). XRD is a useful technique applied herein to characterize the physical state of liraglutide entrapped in PLGA NPs and further to confirm the stability attributed to polymer-drug interaction. Powder X-ray diffraction (XRD) patterns of pure Lira, PLGA, Lira free/loaded PLGA NPs were obtained using an Xray powder diffraction (XRPD) BRUKER D8 Advance X-ray diffractometer (Bruker AXS GmbH, Karlsruhe, Germany), supplied with a $\mathrm{Cu} K \lambda 1$ radiation source $\left(\lambda=1.54056 \mathrm{~A}^{\circ}\right)$, with a voltage of $40 \mathrm{kV}$ and a current of $40 \mathrm{~mA}$, in flat plate $\theta / 2 \theta$ geometry, over the $2 \theta$ ranges $3-40^{\circ}$, with a scan time of $0.1 \mathrm{~s}$ at step size of $0.007^{\circ}$. The sample was placed on a quartz holder and measured at ambient temperature and humidity.

\section{Stability of Lira Encapsulated in PLGA NPs}

Electrospray Ionization Mass Spectrometry. Electrospray Ionization Mass Spectrometry (ESI-MS) is a valuable tool to be used to provide information about the molecular weight of native Lira and compare it to Lira loaded in NPs. Lira was characterized by an Agilent 1100 LC-MSD trap mass spectrometer equipped with an electrospray ion source.

Circular Dichroism. CD was performed to evaluate the conformational stability of Lira loaded into the prepared polymeric NPs. CD spectra were obtained with a Jasco J-1 100 spectropolarimeter (Tokyo, Japan). Aliquot of each of PBS ( $\mathrm{pH}=$ 8.1), native Lira in PBS and Lira extracted from NPs in PBS was placed in a $10 \mathrm{~mm}$ pathway Far-UV quartz cuvette and the Far-UV CD spectra were collected by an PM-539 CD spectrometer. Spectra were collected at room temperature over the wavelength range of $260 \mathrm{~nm}$ to $195 \mathrm{~nm}$ with $0.2 \mathrm{~nm}$ interval. Ellipticity was recorded at scanning speed of $100 \mathrm{~nm} / \mathrm{min}$ and $1.00 \mathrm{~nm}$ band with 5 accumulations. PBS solution subtraction, noise reduction and data analysis were performed using standard analysis and temperature/ wavelength analysis programs (Jasco).

\section{RESULTS AND DISCUSSION}

\section{Placket Burman Design: Risk Analysis}

The QTPP that encompasses the desired CQAs was defined in our previous paper as following: stable, homogeneous and spherical shaped freeze dried NPs with particle size of 100$300 \mathrm{~nm}$ and maximum EE. Risk assessment was also conducted (using LEAN-QbD software) for ranking and prioritizing CMAs and CPPs likely to have an impact on the quality of Lira loaded PLGA NPs (20) and the highly influential parameters were prioritized and subjected to subsequent screening using a seven-factor, two-level, eight-run PB screening design (Table 2) in order to minimize their risk to a low level by controlling these variables in a specific accepted range.

Herein, the possible effects of these formulation and process parameters on four responses namely: mean particle size, PDI, EE and zeta potential were investigated by applying the $\mathrm{PB}$ screening design where the experimental data were validated by ANOVA for each factor. ANOVA parameters for predicting mean particle size $(\mathrm{Y} 1), \mathrm{PDI}(\mathrm{Y} 2), \mathrm{EE}(\mathrm{Y} 3)$ and Zeta potential (Y4) are presented in Supplemental Table 1.Surface response plots are also useful diagrammatic representation of the values of the response, to project the significance of effects for each variable and can explain the relationship between tested independent factors and dependent responses. A color-scale object along with the surface plot serves as a legend, and the value of the response is dependent on the gradual 
Table 2 The Input Factor Levels in 7 Factor, 2 Level, 8 Run PBD

\begin{tabular}{llllllll}
\hline Run code & PLGA $(\mathrm{mg})$ & Lira $(\mathrm{mg})$ & 2nd sonication time $(\mathrm{min})$ & PVA (\%) & Lyoprotectant type & Lyoprotectant (\%) & W $_{2} / \mathrm{O}_{\text {ratio }}$ \\
\hline PBD-FI & 30 & 0.5 & 0.5 & 2 & Trehalose & 10 & 2 \\
PBD-F2 & 60 & 0.5 & 0.5 & 0.5 & Mannitol & 10 & 5 \\
PBD-F3 & 30 & 5 & 0.5 & 0.5 & Trehalose & 5 & 5 \\
PBD-F4 & 60 & 5 & 0.5 & 2 & Mannitol & 5 & 5 \\
PBD-F5 & 30 & 0.5 & 2 & 2 & Mannitol & 5 & 5 \\
PBD-F6 & 60 & 0.5 & 2 & 0.5 & Trehalose & 5 & 10 \\
PBD-F7 & 30 & 5 & 2 & 0.5 & Mannitol & 10 & 2 \\
PBD-F8 & 60 & 5 & 2 & 2 & Trehalose & 5 \\
\hline
\end{tabular}

color. Based on this color gradient, these plots can present the change in response value with different levels of the independent variable.

\section{Influence of Investigated Parameters on the Z-Average Size, PDI, EE and Zeta Potential}

Depending on the selected parameters levels, the Z-average ranged between $160.1 \pm 5.6 \mathrm{~nm}$ and $235 . \pm 5.3$ (Table 3 ). The corresponding coefficients are summarized in Supplemental Table 2, where the factors a $P$ value $<0.05$ are regarded as highly significant, while the ones having nonsignificant response coefficients with a $\mathrm{P}$ value $>0.05$ are least contributing in the prediction of mean particle size. The polynomial equation obtained for the fitted full model explaining the effect of formulation and process variables on the mean particle size is:

$$
\begin{aligned}
\mathrm{Y}_{I=} & \left|97.802 I_{+} 9.5079 \mathrm{X}_{\mid}+\right| .5304 \mathrm{X}_{2}+7.7638 \mathrm{X}_{3} \\
& -\left|6.9754 \mathrm{X}_{4}-5.47\right| 2 \mathrm{X}_{5}-3.5429 \mathrm{X}_{6}-7.079 \mid \mathrm{X}_{7} \\
& \text { with } \mathrm{R}^{2}=0.9745, \text { adjusted } \mathrm{R}^{2}=0.9609 \\
& \text { and Mean square }(\mathrm{MS})=22.3978
\end{aligned}
$$

According to this polynominal equation and the Pareto chart (Fig. 1), the most influential factors in terms of particle size are the PVA concentration, PLGA amount followed by sonication time and $\mathrm{W} 2 / \mathrm{O}$ ratio at almost the same level of significance. Then the next important variables include lyoprotectant type and concentration. The tested levels of Lira amount were observed to have a non-statistically significant effect on the mean particle size.

In all prepared formulations, NPs exhibited a practically monodisperse or narrow distribution (21) as PDI was ranged from $0.1 \pm 0.003$ in PBD-F6 to $0.22 \pm 0.01$ in PBD-F3 (Table 3) evidencing that the obtained NPs are homogeneous and stable with no aggregation. Figure $\mathrm{S} 1$ shows an example of PDI $=0.07$ with $\mathrm{Z}$-average $=157.1 \mathrm{~nm}$ obtained for PBDF1. The polynomial equation obtained for the full model describing the effect of formulation and process variables on the PDI value is:

$$
\begin{aligned}
\mathrm{Y} 2= & 0 .|568-0.006| \mathrm{X}|+0.03| 2 \mathrm{X} 2+0.0005 \mathrm{X} 3 \\
& -0.00|3 \mathrm{X} 4-0.0032 \mathrm{X} 5-0.003| \mathrm{X} 6+0.029 \mid \mathrm{X} 7 \\
& \text { with } \mathrm{R}^{2}=0.9235, \text { adjusted } \mathrm{R}^{2}=0.8828, \\
& \text { and Mean square }(\mathrm{MS})=0.0003 \\
\mathrm{Y} 2= & 0 .|568-0.006| \mathrm{X}|+0.03| 2 \mathrm{X} 2-0.0032 \mathrm{X} 5 \\
& -0.003|\mathrm{X} 6+0.029| \mathrm{X} 7 \ldots \ldots . \text { Redcuced}(\text { model }) \\
& \text { with } \mathrm{R}^{2}=0.92256, \text { adjusted } \mathrm{R}^{2}=0.89522, \text { and } \\
& \text { Mean square }(\mathrm{MS})=0.0002 .
\end{aligned}
$$

The statistical analysis (Table 3S) along with the Pareto chart (Fig. 3) revealed that only two of the examined CMAs namely Lira amount and $\mathrm{W}_{2} / \mathrm{O}$ ratio were observed to have a significant effect on the PDI value.

Depending on the tested two levels of each factor, the results showed that EE varied between $20.1 \pm 1.7$ in formulation PBD-F1 and 43.5 \pm 3.3in PBD-F4 (Table 3).The polynomial equation obtained for the fitted full model showing the impact of the seven examined variables on $\mathrm{EE}$ is:

Table 3 Experimental Responses Results in PBD

\begin{tabular}{lllll}
\hline Run code & $\begin{array}{l}\text { Z-AVE } \\
(\mathrm{nm})\end{array}$ & PDI & EE\% & $\begin{array}{l}\text { Z-potential } \\
(\mathrm{mV})\end{array}$ \\
\hline PBD-FI & $160.1 \pm 5.6$ & $0.10 \pm 0.03$ & $20.1 \pm 1.7$ & $-30.6 \pm 1.8$ \\
PBD-F2 & $209.8 \pm 8.01$ & $0.15 \pm 0.01$ & $36 \pm 1.25$ & $-25 \pm 1.6$ \\
PBD-F3 & $190.0 \pm 2.8$ & $0.23 \pm 0.01$ & $41 \pm 2.46$ & $-27.3 \pm 0.7$ \\
PBD-F4 & $200.2 \pm 3.5$ & $0.16 \pm 0.03$ & $43.5 \pm 3.34$ & $-31.2 \pm 1.2$ \\
PBD-F5 & $179.5 \pm 3.8$ & $0.17 \pm 0.01$ & $32 \pm 4.03$ & $-23.8 \pm 1$ \\
PBD-F6 & $235.7 \pm 5.3$ & $0.10 \pm 0.003$ & $28.9 \pm 2.05$ & $-29.2 \pm 0.4$ \\
PBD-F7 & $223.6 \pm 3.7$ & $0.17 \pm 0.02$ & $22.2 \pm 2.12$ & $-30.4 \pm 0$. \\
PBD-F8 & $183.5 \pm 3.03$ & $0.20 \pm 0.01$ & $21 \pm 1.51$ & $-26 \pm 0.2$ \\
\hline
\end{tabular}



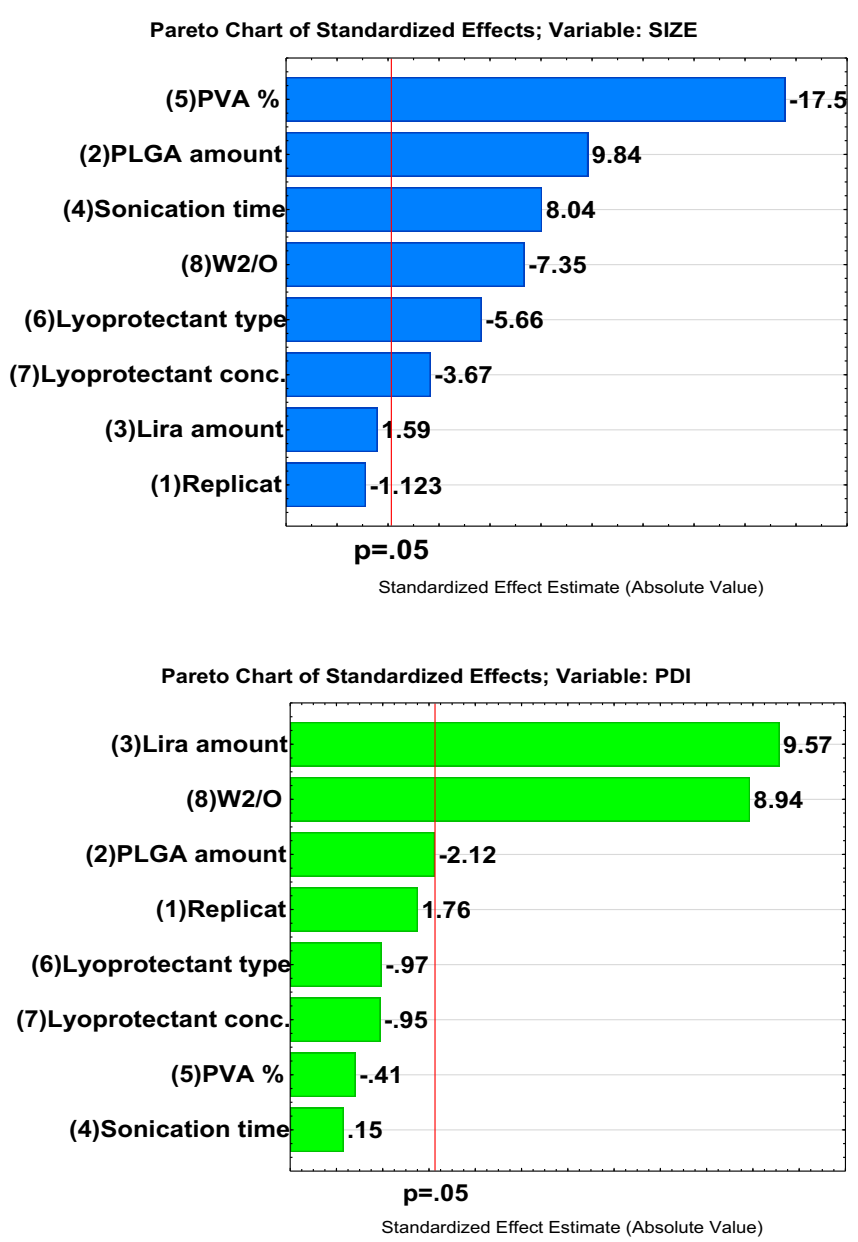

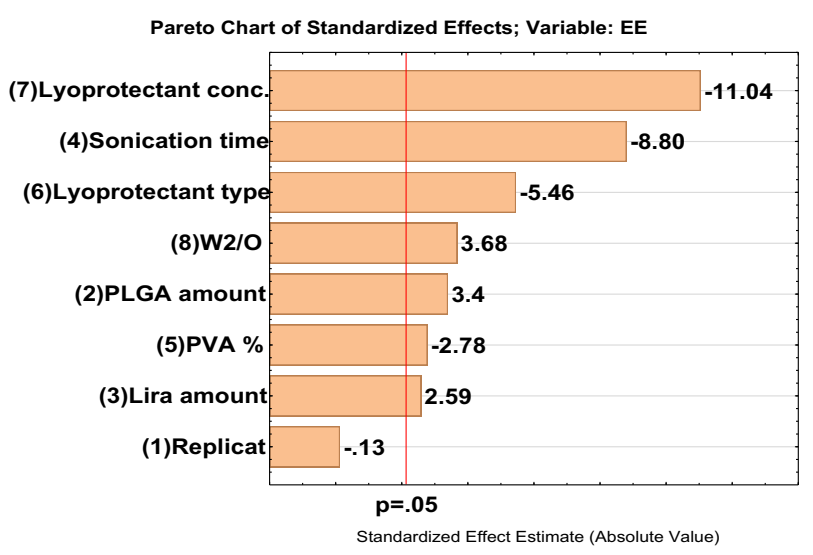

Pareto Chart of Standardized Effects; Variable: ZETA POTENTIAL

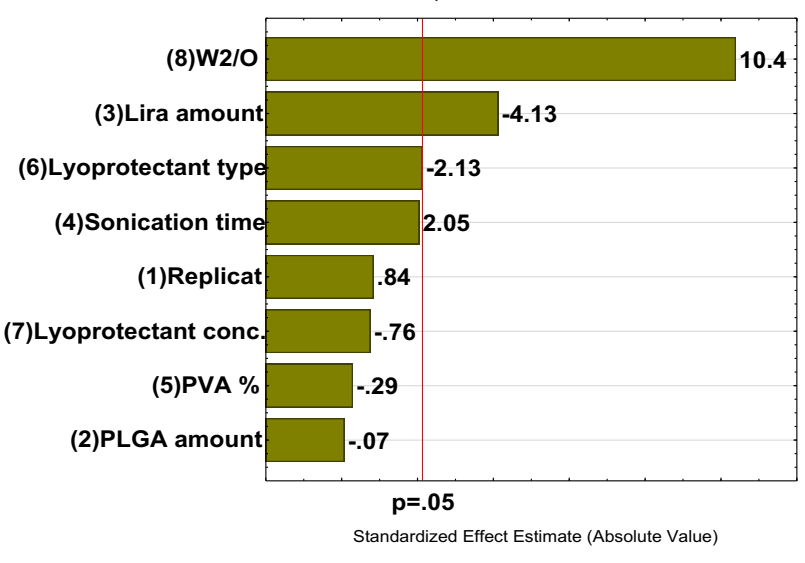

Fig. I Pareto charts of the effects of the examined independent variables on Z-average size (YI), PDI (Y2), EE (Y3), zeta potential (Y4). Replicate refers to number of repetitions for each formula which was 3.

$$
\begin{aligned}
\mathrm{Y} 3= & 30.548+1.7683 \mathrm{X}_{1}+1.3458 \mathrm{X}_{2}-4.5825 \mathrm{X}_{3} \\
& -1.4458 \mathrm{X}_{4}-2.8408 \mathrm{X}_{5}-5.7483 \mathrm{X}_{6}+\mid .915 \mathrm{X}_{7} \\
& \text { with } \mathrm{R}^{2=} 0.947 \mid, \text { adjusted } \mathrm{R}^{2}=0.9 \mid 89 \\
& \text { and Mean square }(\mathrm{MS})=6.502 \mid .
\end{aligned}
$$

This equation along with the Pareto chart (Fig. 1) and statistical analysis (Table $4 \mathrm{~S}$ ) show that the lyoprotectant \%, 2nd sonication time and lyoprotectant type are the most highly risky factors in terms of EE. This is followed by other factors which all show a significant impact on the amount of Lira encapsulated in the PLGA NPs.

The zeta potential was also monitored during the optimization steps and its values ranged from-31.2 $\pm 1.2 \mathrm{mV}$ in PBD-F4 to $-23.8 \pm 0.95 \mathrm{mV}$ in PBD-F5 (Table 3 ), and these expected negative values are attributed to the presence of carboxyl group end on PLGA. Figure S2 depicts the result of zeta potential obtained for formulation PBD-F1 as an example. The full model describing the effect of formulation and process variables on the zeta potential is:

$$
\begin{aligned}
\mathrm{Y}_{4=}- & 28.0596-0.0154 \mathrm{X}_{1}-0.907 \mid \mathrm{X}_{2}+0.4496 \mathrm{X}_{3} \\
& -0.0646 \mathrm{X}_{4}-0.4679 \mathrm{X}_{5}-0.1663 \mathrm{X}_{6}+2.2846 \mathrm{X}_{7} \\
& \text { with } \mathrm{R} 2=0.9001, \text { adjusted } \mathrm{R} 2=0.8468, \\
& \text { and Mean square }(\mathrm{MS})=1.1599 \\
\mathrm{Y} 4= & -28.0596-0.907 \mid \mathrm{X} 2+0.4496 \mathrm{X} 3-0.4679 \mathrm{X} 5 \\
& -0.1663 \mathrm{X} 6+2.2846 \mathrm{X} 7 \ldots \ldots \ldots \text { Reduced (model) } \\
& \text { with } \mathrm{R} 2=0.89946, \text { adjusted } \mathrm{R} 2=0.86397, \\
& \text { and Mean square }(\mathrm{MS})=1.0 .297 .
\end{aligned}
$$

It is obvious from the statistical analysis (Table $5 \mathrm{~S}$ ) and the Pareto chart (Fig. 1) that only the $\mathrm{W} 2 / \mathrm{O}$ ratio and the lira amount had a significant impact $(P<0.05)$ on the surface charge of PLGA NPs. Other examined variables were observed to have only a non-significant effect on surface charge.

The effect of the above-explained variables on $\mathrm{Y} 1, \mathrm{Y} 2, \mathrm{Y} 3$ and $\mathrm{Y} 4$ is discussed point by point in the following: 


\section{Effect of Polymer Amount}

It is apparent from Fig. 2 that when the PLGA amount was increased, the Z-average increased correspondingly, as supported by many earlier published papers $(22,23)$ That could be explained by increasing the viscosity of the organic phase which leads to a reduction in the net shear stress (24), in addition to a reduction in the evaporation rate; i.e. the dispersion rate of the organic phase toward the external aqueous phase will be slower, thus that incites the formation of larger particles $(25,26)$. The formation of a more viscous organic phase was reported to push up the frequency of collisions between particles during the emulsification and droplet solidification step, which may lead to the aggregation of the semisolid particles (5). Regarding the PDI value, it was also observed that size distribution was slightly decreased by increasing the PLGA amount which means that a greater level of PLGA would promote the formation of much more homogeneous NPs.

The positive effect that the PLGA amount has on EE (Fig. 4) could be again due to the fact of increasing the viscosity of the organic phase with a higher amount of polymer, which can retard drug diffusion into the external aqueous phase and thus increase the amount of drug entrapped inside the NPs $(27,28)$. It is also known that larger nanoparticles obtained with a higher PLGA level can provide sufficient surface for entrapping the peptide drug. Furthermore, higher PLGA levels give rise to more rapid polymer deposition as ethyl acetate is removed from the NPs, which is expected to hinder any undesirable Lira diffusion into the external phase (29).

\section{Effect of Liraglutide Amount}

The positive effect that Lira has on the 2nd emulsification and both final particle size (Fig. 2) and size distribution as a result (Fig. 3) would be explained by the influence of the drug on the droplet size of the inner aqueous phase within the organic phase in the first water-in-oil (wl/o) emulsion, which may modify its ability for dispersion in the outer aqueous phase. However, this effect was only limited and not significant in the case of particle size, while Lira theoretical loading amount was a significant influential parameter affecting the PDI and zeta potential values (Fig. 1, Fig. 3, Fig. 5). The Lira level was also shown to have a significant positive effect on EE; when loading higher amount of Lira, EE was even higher (Fig. 4). This positive trend was previously reported with other peptide drugs as insulin, and it is explained due to higher amount of peptide that is associated with the surface of nanoparticles and is electrooptically linked to a greater extent (30), thus resulting in a higher EE value. However, the studied levels of the Lira amount in our work were shown to be the least risky factor affecting $\mathrm{EE}$ as presented in the Pareto chart (Fig. 1).
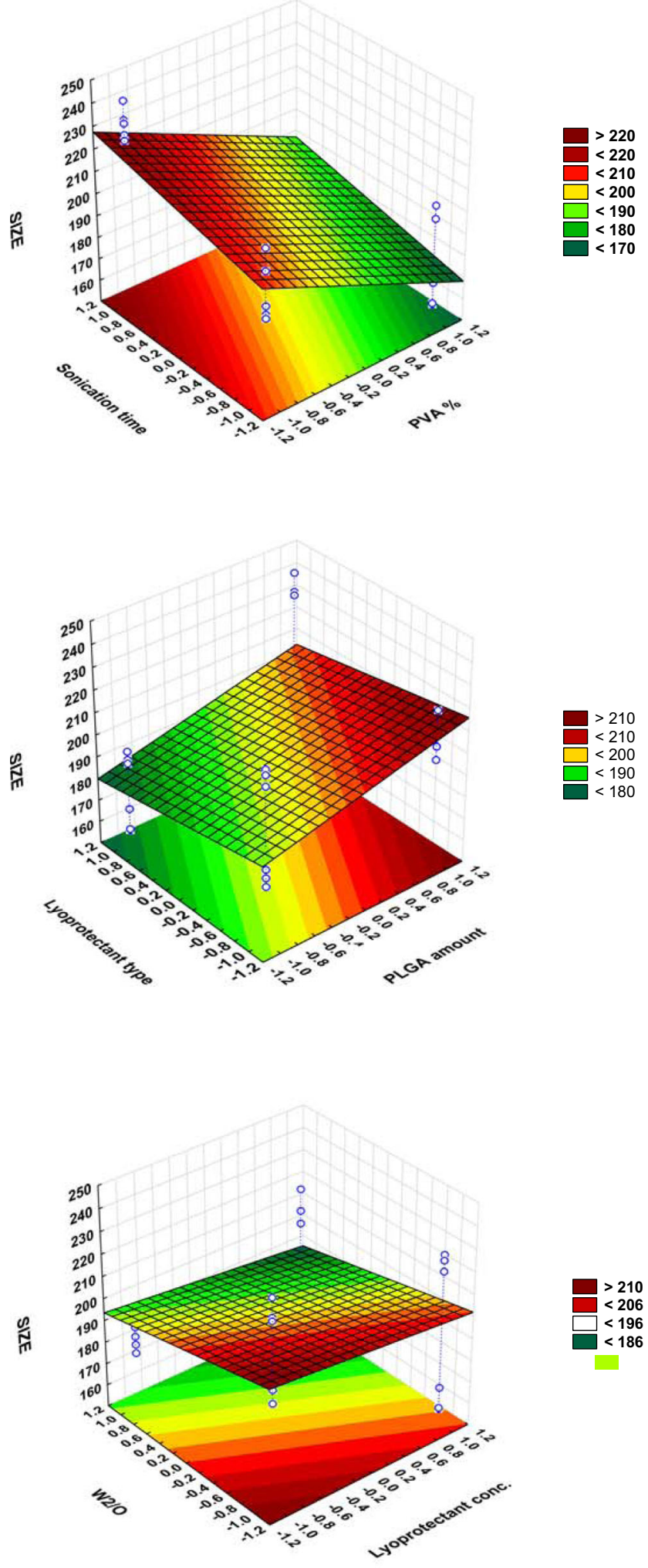

Fig. 2 Surface plots showing the effect of the significant examined variables on the Z-average size $(\mathrm{YI})$. 
Fig. 3 Surface plot showing the effect of lira amount and $W_{2} / O$ ratio on PDI (Y2).

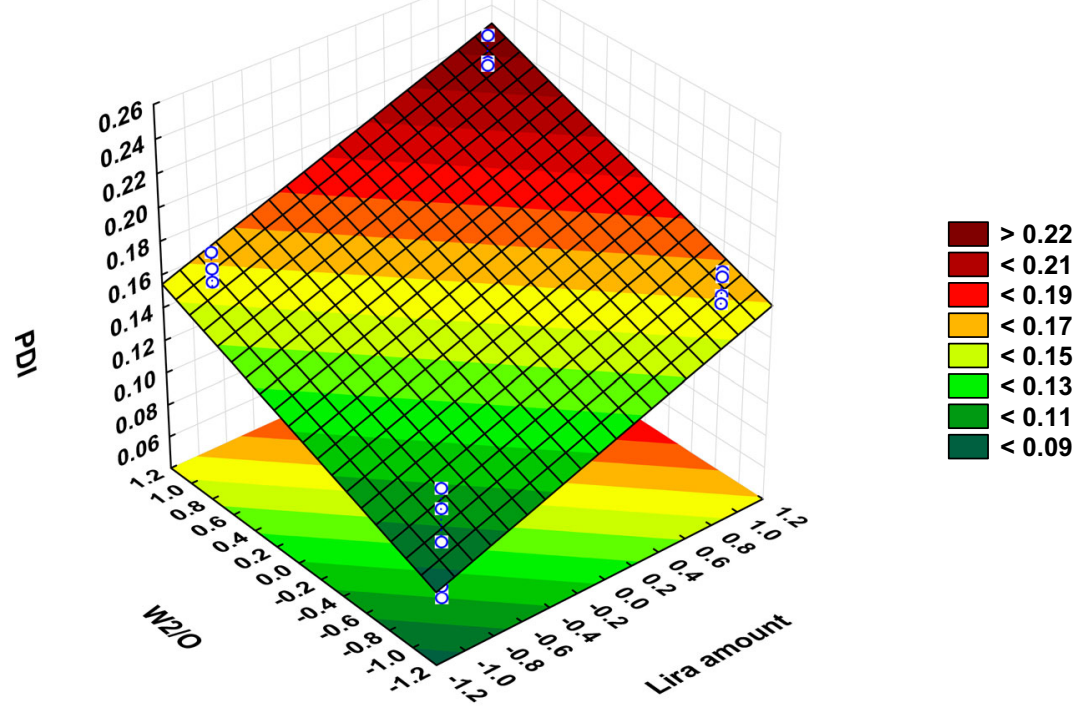

\section{Effect of PVA Concentration}

PVA has previously proved to be a good choice as a surfactant used to prepare stable PLGA NPs with a small size and a narrow PDI due to its ability of minimizing the surface tension of the continuous phase which is an aqueous phase in our work. The statistical analysis of the results showed that increasing PVA level played a crucial role in decreasing particle size

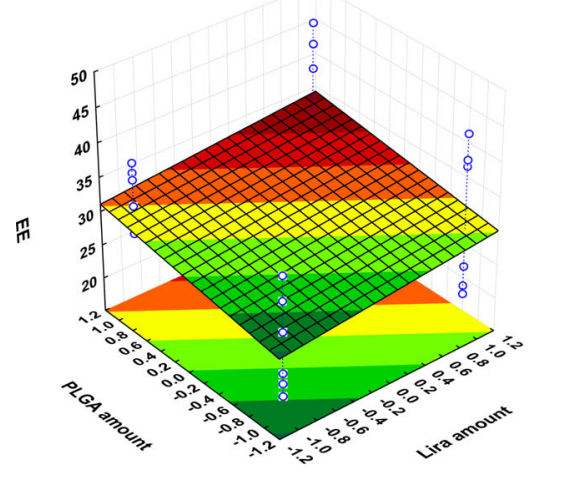

$\square^{>30}<27$
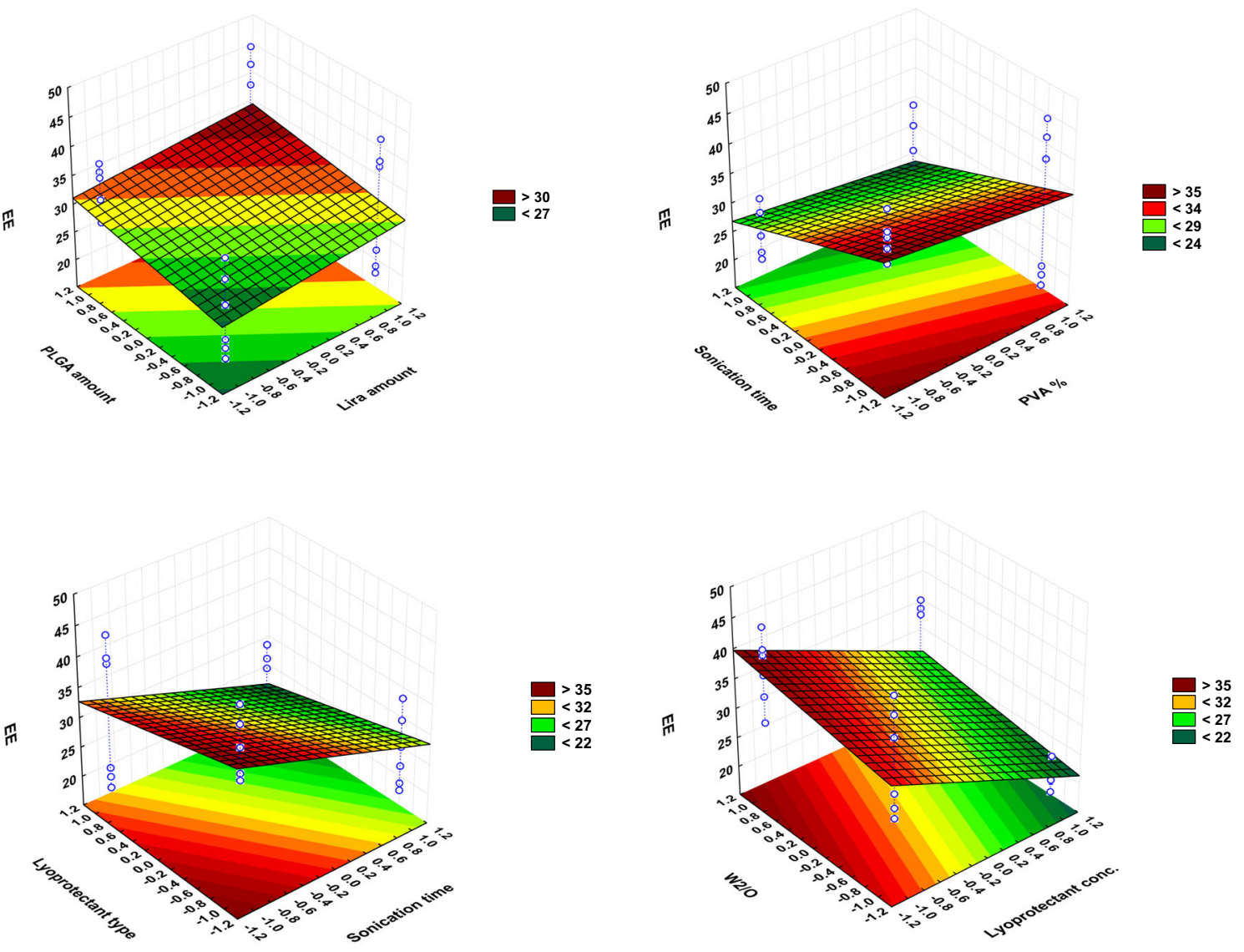

Fig. 4 Surface plot showing the effect of examined variables on EE (Y3). 
Fig. 5 Surface plot showing the effect of lira amount and $W_{2} / O$ ratio on zeta potential (Y3).

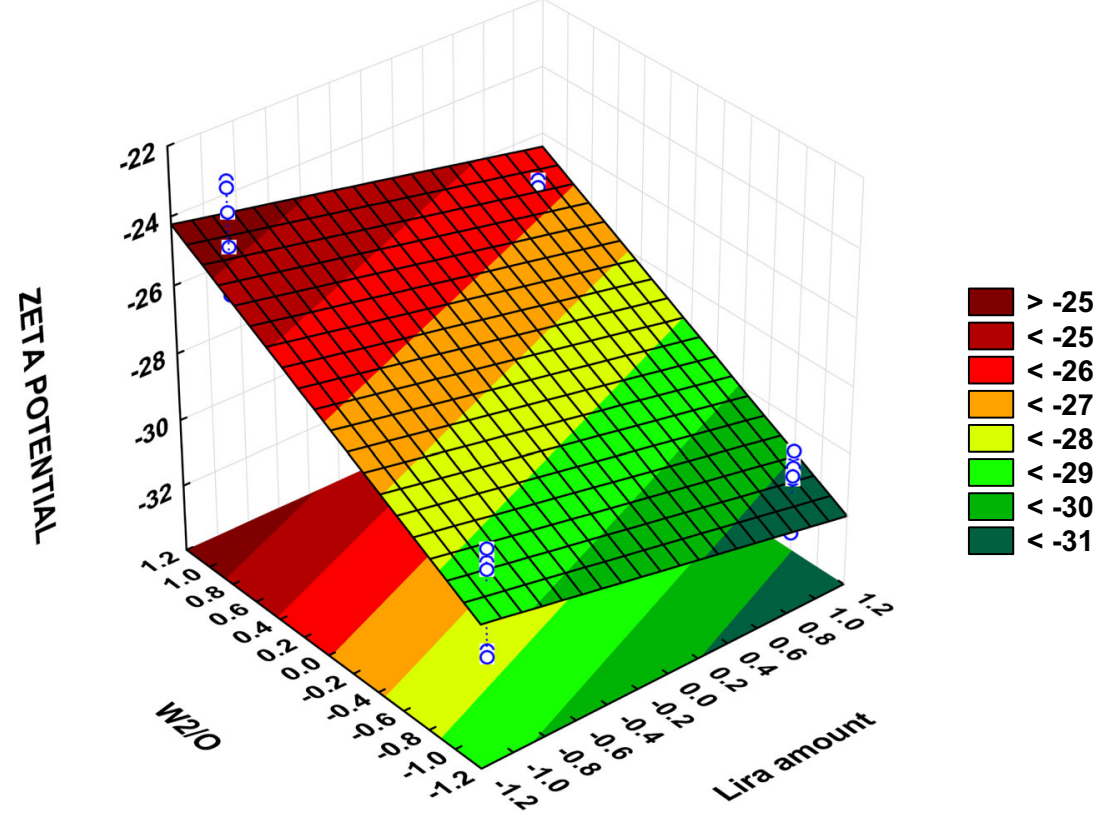

(Fig. 2), and it was the leading factor impacting the size of NPs as can be seen in the Pareto chart (Fig. 1) which is in agreement with previous reports $(31,32)$. This result can be expected from the stabilizing function of PVA molecules that tend to align themselves at the droplet surface lowering the free energy at the interface between two phases and avoiding coalescence between nanodroplets, thus stabilizing the smaller droplets and preventing coalescence into a larger one $(33,34)$. Hence, at a low PVA concentration, a larger particle size was obtained due to insufficient reduction in interfacial tension. It

Table 4 The Observed and the Predicted Values of the Response Values of the Center Checkpoints

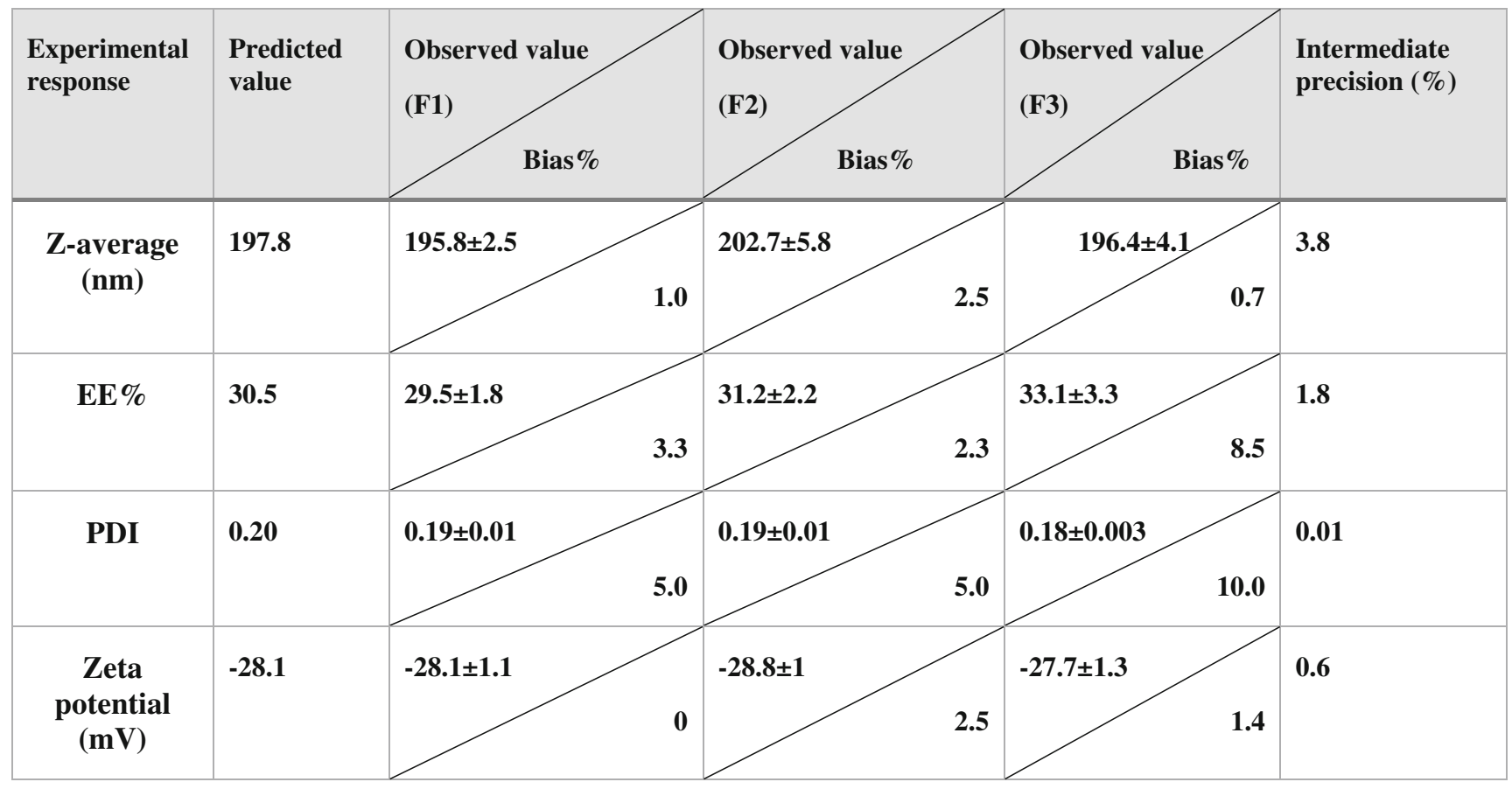


was also reported that a fraction of PVA remains associated with the surface of nanoparticles even after the washing of nanoparticles (33). Thus, the presence of the PVA layer on the surface of nanoparticles may also improve their stability during the freeze-drying process.

It is also clear that as PVA level increased from $0.5 \%$ to $2 \%$, EE decreased (Fig. 4). A possible explanation of this negative impact was discussed in a preceding published work, as it was proved that the breakdown of the inner aqueous droplets containing Lira took place along with the fragmentation of the organic phase because of the cavitation occurs in the complex system of three phases, the higher level of PVA in the external aqueous phase is attributed to enhancing the breakdown of inner aqueous droplets and a higher amount of Lira can escape to the external phase as a result (34). This is supported by a previous paper in which increasing the emulsifier concentration had led to lower entrapment of the protein drug in PLGA NPs, which was explained as a result of increasing the partitioning of the drug from the inner to the outer phase (35).

\section{Effect of 2nd Sonication Time}

The results revealed that larger and less homogeneous particles were yielded when increasing 2 nd sonication time from $30 \mathrm{~s}$ to 2 min (Fig. 1, Fig. 2). This could be explained as follows: at the beginning, increasing the sonication time led to the formation of smaller droplets due to the production of higher energy and higher shearing rates, which are more efficient in breaking large droplets into smaller ones (36). However, a further elevation in this sonication period resulted in the re-aggregation of these particles. This trend is in accordance with results obtained by others where they observed the formation of larger droplets as an outcome of longer sonication or homogenization time (37). Besides, the longer the 2nd sonication time, the higher the shear energy input, thus the higher the leached amount of peptide from $\mathrm{W} 1 / \mathrm{O}$ to the external aqueous phase i.e. the lower the EE. The Pareto chart (Fig. 1) demonstrates that the prolonged 2nd sonication time was the 2nd highest risky factor regarding the influence on EE.

\section{Effect of Lyoprotectant Type and Concentration}

Lyoprotectant are commonly used to stabilize the particles and protect them from degradation during freeze-drying and storage (38). Regarding the type of lyoprotectant used in this study, these significant changes in particle size may be related with the behavior of each lyoprotectant during freeze-drying, and the adsorption of lyoprotectant on the surface of nanoparticles. It is clear that trehalose is more effective in obtaining smaller nanoparticles (Fig. 1, Fig. 2).This is in accordance with previous papers that confirmed that trehalose which is a non-reducing sugar could be the most preferable lyoprotectant of choice because of its merits over the other sugars; including a very low chemical reactivity, a higher glass transition temperature $\mathrm{Tg}$, less hygroscopicity, in addition to the absence of internal hydrogen bounds, allowing a more flexible formation of hydrogen bonds with nanoparticles during the freeze-drying process (39). However, mannitol was proved to be more effective in obtaining a higher EE value according to our experimental work. Trehalose was investigated before regarding its effect on the secondary structure of insulin, and the results showed that it highly affected the conformational stability of the peptide; so it might not be the best choice to encapsulate peptide drugs (40). In addition to that; mannitol is able to form crystal morphology (which is confirmed later in this paper by DSC and XRD) and this might be attributed to the stability of peptide.

When it comes to the lyoprotectant level, results revealed that increasing this level up to $10 \%$ significantly reduced the Z-average and slightly minimized the PDI of the obtained NPs (Fig. 1,Fig. 2), which means that at this level the used lyoprotectants are more efficient in preventing the aggregation and stabilizing the PLGA NPs the use of an excess amount of lyoprotectant might eventually make it reach the limit of its stabilization ability and thus the agglomeration of NPs is likely to increase (41). It is also apparent from the statistical analysis presented in the Pareto chart and surface plot (Fig. 1, Fig. 4) that the lyoprotectant level was the most influential formulation variable impacting EE. When the level of lyoprotectant continued increasing, the amount of entrapped Lira significantly decreased, which could be the result of smaller NPs obtained with a higher lyoprotectant level, and thus less sufficient surface area for entrapping the drug.

\section{Effect of $\mathrm{W}_{2} / \mathrm{O}$ Ratio}

As the volume ratio of external aqueous phase to organic phase $\mathrm{W} 2 / \mathrm{O}$ went on increasing, which was achieved by increasing the volume of the external aqueous phase, the average particle size was significantly decreased as can be seen from the Pareto chart and surface plot (Fig. 1, Fig. 2). This formation of smaller droplets may be due to the higher amount of stabilizer present as compared to the nonsufficient amount of stabilizer when using a lower amount of this phase. It was also recently reported that increasing the continuous phase volume:organic phase ratio had led to particle size reduction (27).

Regarding PDI values, a significant increase in size distribution was observed when the volume of the external aqueous phase was higher, as shown by the Pareto chart and surface plot (Fig. 1, Fig. 3). This observation might be attributed to a reduction of shear stress during the homogenization process 

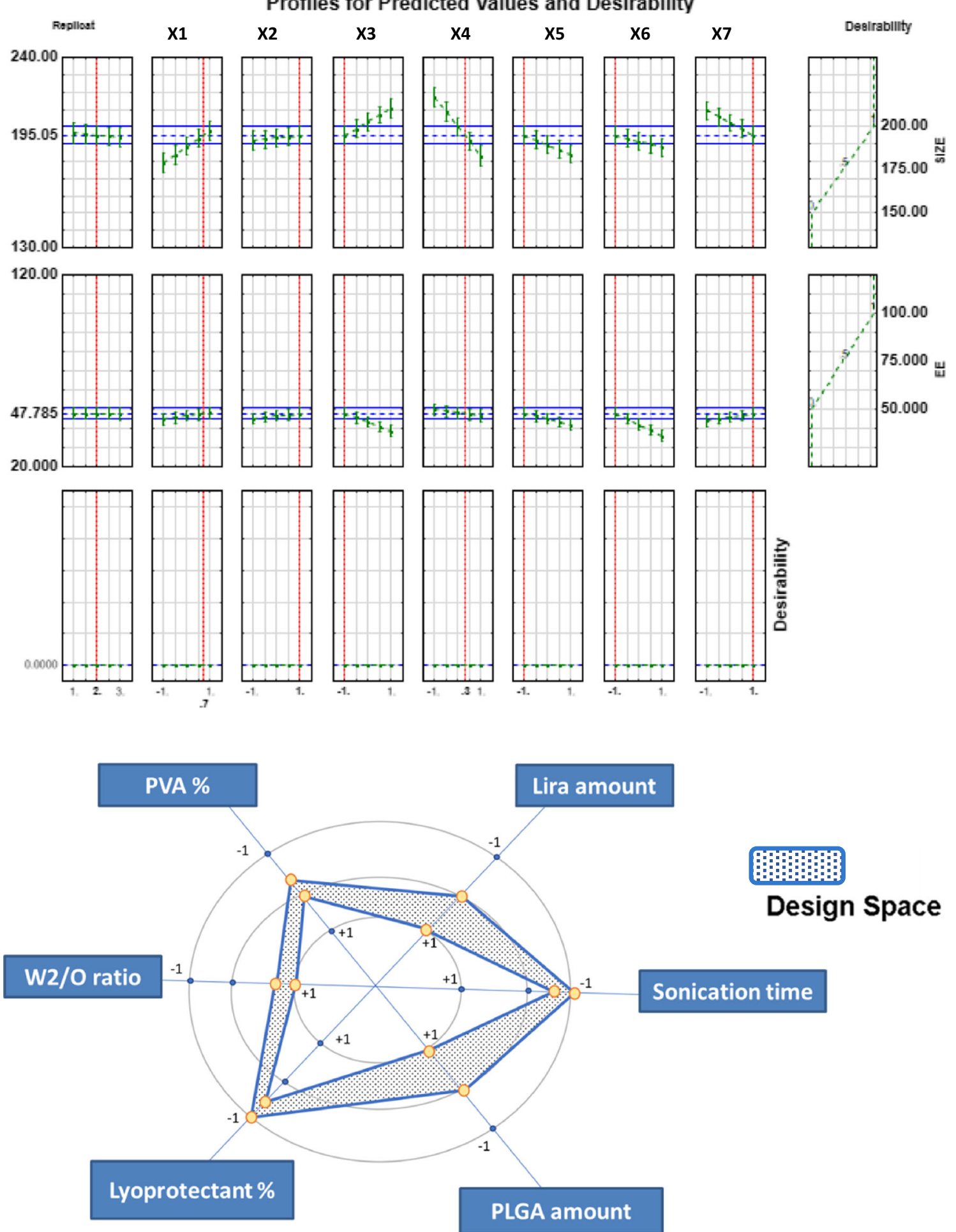

Fig. 6 The desirability plots and graphical design space representing the optimum levels of factors required to prepare the optimized formula.

(42). Besides, the phase ratio was the highly influential factor affecting the surface charge as increasing the external aqueous phase volume led to a significant increase in the zeta potential value (Fig. 5).
The EE exhibited a significant upward trend when increasing the volume of the external aqueous phase, as presented in the Pareto chart and surface plot (Fig. 1,Fig. 4). The impact of the external aqueous phase/organic phase is controversial, as 
Table 5 The Observed and the Predicted Values of the response values of the Optimum Lira Nanoparticle

\begin{tabular}{lllll}
\hline Experimental response & Predicted value & Observed value & Residual & Bias (\%) \\
\hline Z-average $(\mathrm{nm})$ & 197.9 & $189 \pm 4.99$ & 8.95 & 4.5 \\
EE\% & 48.3 & $51.8 \pm 2.39$ & 3.5 & 7.2 \\
PDI & 0.21 & $0.19 \pm 0.012$ & 0.034 & 7.8 \\
Zeta potential $(\mathrm{mV})$ & -26.5 & $-27.1 \pm 1.33$ & 0.58 & 2.2 \\
\hline
\end{tabular}

many papers reported that increasing the $\mathrm{W} 2 / \mathrm{O}$ ratio can lead to minimizing the amount of the encapsulated drug $(31,42)$. However, other published papers assumed that a relatively higher volume ratio of the external aqueous phase was beneficial for maximizing the drug encapsulated in the NPs as a higher outer aqueous phase volume can speed up the solidification time (evaporation of ethyl acetate and formation of NPs), while the smaller the volume of this outer aqueous phase, the longer the time required for solidification, thus over this time Lira may leak to the outer phase due to its hydrophilicity (43).
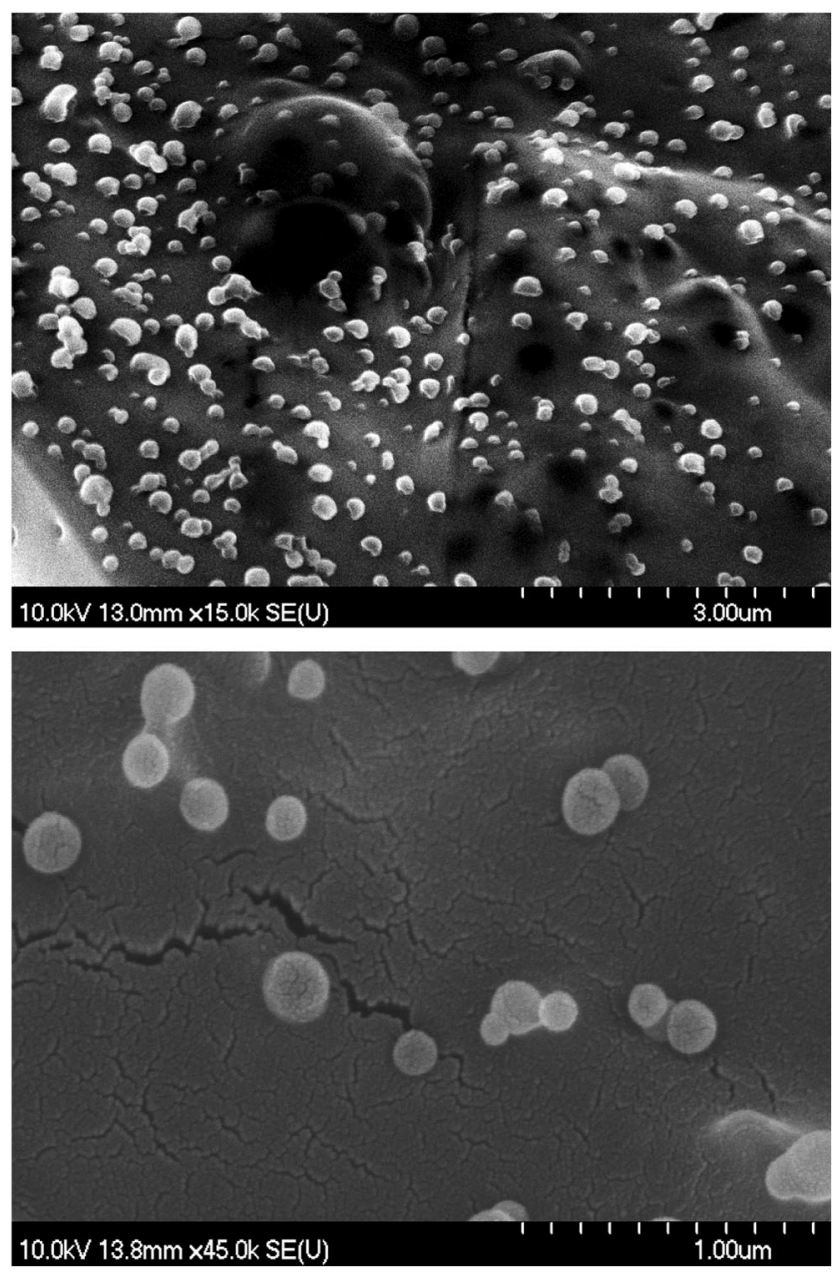

Fig. 7 SEM images of liraglutide loaded PLGA NPS.

\section{Placket Burman Design: Model Validation}

The three replications of center checkpoint formulations were prepared and evaluated for the particle size, EE, PDI and zeta potential to evaluate the reproducibility of the generated models and estimate the experimental error. Table 4 presents the percentage of bias between predicted and observed values for each response was calculated by means of the following equation .

Bias $(\%)=($ Predicted value - observed value $) /$ Predicted value ${ }^{*} \mid 00$

The minor differences between the predicted values and the average of experimental values confirm the validity of this design in providing a good prediction of the four tested responses.

In addition to that, the calculated relative standard deviation $\mathrm{RSD}^{\%}$ values that are presented in Table 4 prove the repeatability and intermediate precision regarding the 4 responses that further confirms high analytical process variability.

\section{Placket Burman Design: Design Space and Optimization}

After establishing the polynomial equations describing the relationship between the CPPs, CMAs and the examined responses namely; particle size, EE, PDI and zeta potential, the optimization process was conducted. Among the four responses, size and EE were the highly critical quality attributes of nanoparticles being significantly affected by almost all the tested variables which is in accordance with the estimated severity scores of CQAs that was calculated previously at the initial risk assessment process. Therefore, the deign space (DS) was optimized (Fig. 6) targeting the following criteria: the particle size was minimized, encapsulation efficiency was maximized while PDI and Zeta potential were excluded. Thanks to the knowledge obtained via the DS, the optimum levels of the formulation factors were determined: $60 \mathrm{mg}$ of PLGA, $5 \mathrm{mg}$ of Lira, $0.5 \mathrm{~min} 2$ nd sonication time, $1.48 \%$ of PVA, $5 \%$ of mannitol and $\mathrm{W} 2 / \mathrm{O}$ ratio of 5 .As shown in Table 5 , the observed values were comparable to the predicted ones, presenting another confirmation of the validity of the generated 

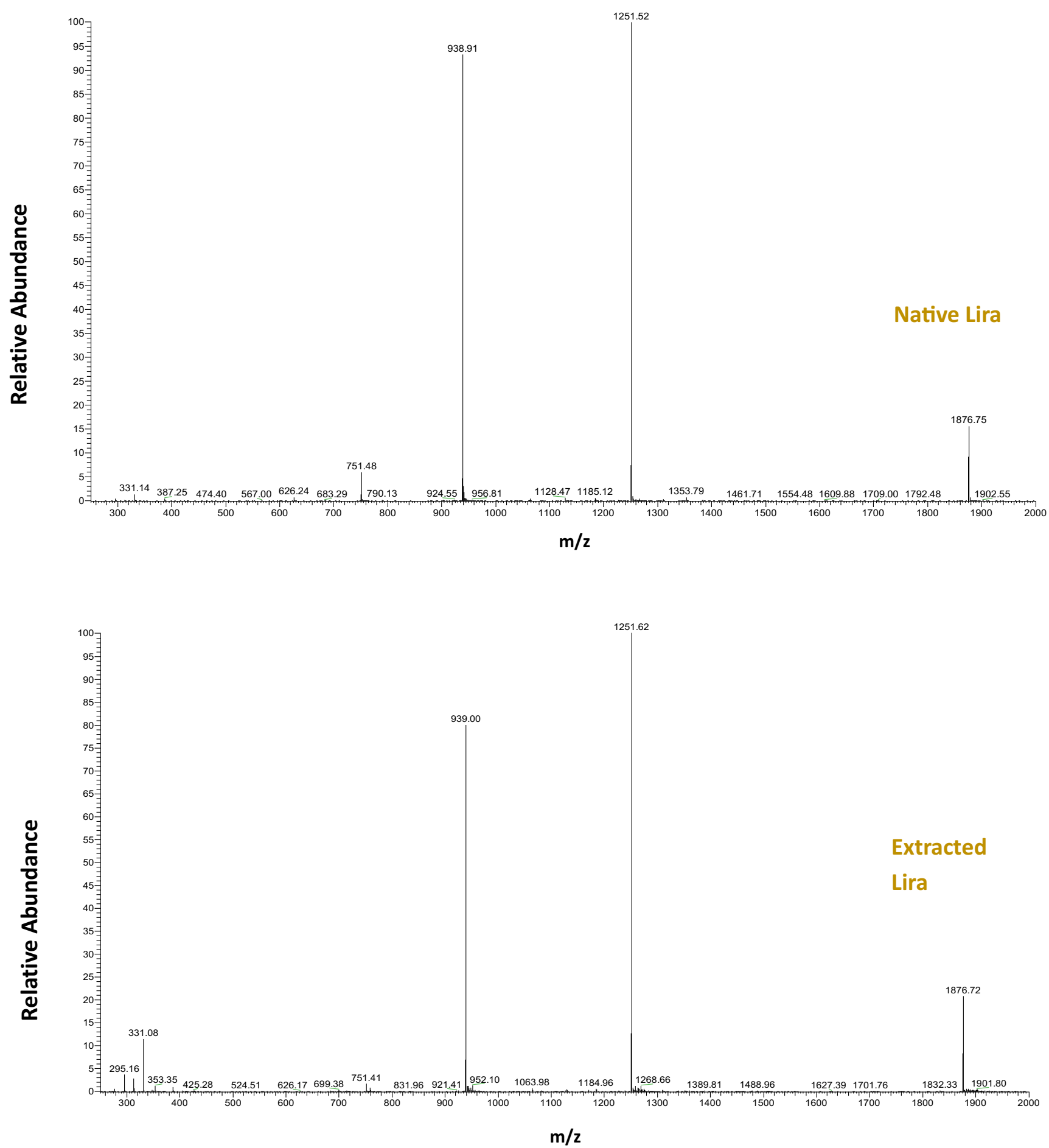

Fig. 8 Mass spectra of lira extracted from PLGA NPs compared to native lira.

models and indicating that the optimized formulation is reliable.

\section{Scanning Electron Microscopy (SEM)}

Figure 7 depicts the shape and surface morphology of the optimized Lira loaded PLGA NPs visualized by SEM. Since the optimized formula was homogeneous (in accordance with low PDI) we selected two images as representative for the sample. The results revealed that Lira loaded PLGA NPs were spherical with quite a smooth surface and they had homogeneous distribution which is in agreement with the abovementioned results that demonstrated low PDI values for all formulations. 


\section{Compatibility Studies}

\section{Fourier Transform Infrared Spectroscopy (FTIR)}

Figure S3. represents the FTIR spectra of pure Lira, pure PLGA and Lira free/loaded PLGA NPs.

The amide I region $\left(1710-1590 \mathrm{~cm}^{-1}\right)$ is the most representative region of the spectra to assess peptide or protein based drug secondary structure (44). In the FTIR of pure Lira, the amide I band was located at $1655 \mathrm{~cm}^{-1}$ and was assigned to $\mathrm{C}=\mathrm{O}$ stretching, while the amide II band was observed at $1541 \mathrm{~cm}^{-1}$ (in-plane $\mathrm{N}-\mathrm{H}$ bending component and $\mathrm{C}-\mathrm{N}$ stretching bands of the amide bond). Besides, the typical peak at $2928 \mathrm{~cm}^{-1}$ was ascribed to C-H stretching of CH3, and the peak at $1396 \mathrm{~cm}^{-1}$ is attributed to amide III. When analyzing the FTIR spectra of Lira loaded PLGA NPs, it was found that the major peaks of pure Lira assigned to amide I and II and III were masked by the PLGA bands, and this seems to be a logical result since the amount of PLGA was much higher than the Lira amount in these NPs. There were no clear differences between the spectra of the blank NPs and Lira loaded NPs which is also expected as the drug loading is very small when compared to the polymer amount. These observations suggest that Lira was successfully loaded into the PLGA NPs.

\section{Differential Scanning Calorimetry (DSC)}

Figure S4 represents the DSC thermograms for pure Lira, PLGA, liraglutide free/loaded PLGA NPs. For the temperature range examined, the PLGA thermogram exhibited a glass transition point at $49.22^{\circ} \mathrm{C}$ and no melting endothermic peak was observed, as PLGA appears amorphous in nature. The DSC thermogram of pure Lira revealed a peak at $275.46^{\circ} \mathrm{C}$, which is attributed to the thermal degradation of this peptide drug, and no endothermic peak of melting was shown, which proved the amorphous nature of the drug. Since the thermogram of Lira loaded PLGA NPs did not display any extra endo/exothermic peaks compared to the blank NPs, this is an indicator of the presence of Lira in the amorphous phase and this drug is successfully encapsulated into the PLGA matrix.

\section{X-Ray Diffraction Study (XRD)}

XRD studies further verified the amorphous nature of both PLGA and pure Lira as they showed no characteristic peaks in their diffractograms which is in accordance with the results of DSC thermograms (Fig. S4). As depicted in Fig. S5, mannitol remained in crystalline state after freeze drying which is due to the property of mannitol to recrystallize at low cooling rates rather than rapid cooling. The crystallization of this lyoprotectant could have a negative effect on the stability of NPs as it is able to limit the formation of these hydrogen bonds (30), and this can explain why trehalose was more efficient than mannitol at preventing the aggregation of NPs and thus minimizing the Zaverage. There was no difference between the diffractograms of the loaded and blank PLGA NPs which is explained in literature as a result of the successful encapsulation of the peptide drug inside the polymeric nanoparticles without change in its physical state (45), and this is in accordance with the DSG results.

\section{Structural Stability}

\section{ESI-MS}

MS was used to compare the molecular weight MW of lira standard (native) to lira extracted from PLGA NPs. As the spectra in Fig. 8 depicts, the measured MW of native Lira and Lira loaded in NPs are almost equal, and the spectra confirmed the presence of Lira with a molar mass of 3751 Da which is an evidence of the integrity of Lira loaded in PLGA NPs prepared using the optimized formulation and process parameters.

Fig. 9 CD spectra of lira extracted from PLGA NPs compared to native lira.

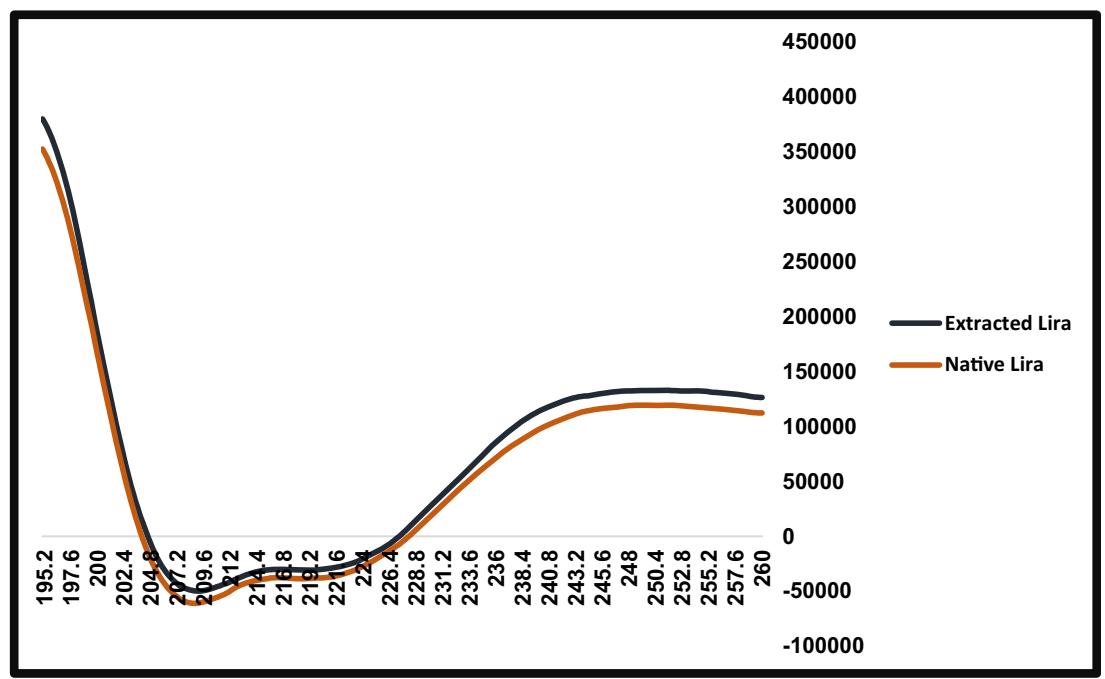




\section{$C D$}

Since the preservation of the secondary structural integrity of a peptide drug in the nanocarrier is critical for its biological efficacy, the secondary structure of Lira extracted from NPs was compared to that of native Lira. The CD spectra of native Lira (Fig. 9) showed two minima at $208.8 \mathrm{~nm}$ and $218.4 \mathrm{~nm}$ indicating the presence of alpha helix elements in the structure, which is in consistent with previous studies on the typical structure of the glucagon-like peptide-1 family. No significant conformational change was recorded for Lira extracted from PLGA NPs (in PBS, $\mathrm{pH}=8.1$ ) as the far UV CD spectra for it showed two minima at $209.4 \mathrm{~nm}$ and $219.2 \mathrm{~nm}$, and almost entirely overlapped with the GD spectrum for the standard.

\section{CONCLUSION AND FUTURE PERSPECTIVES}

The present study is the first published work that substantiated the application of rational QbD-based methodology for the optimization of a GLP-1 analog loaded nanocarrier system. This work demonstrated the importance of implementing DOE within QbD philosophy in the early stage of Liraglutide containing NPs development due to the complexity of this system.

After establishing the design space, with the minimum particle size and maximum EE, the optimized formula was successfully prepared meeting the targeted CQAs. This optimized Lira loaded PLGA NPs formula was also successful in maintaining the native structure of Lira and could be promising for the oral delivery. Thus, in vitro release kinetics, cytotoxicity, intestinal permeability and in vivo studies will be further conducted on this formula.

\section{ACKNOWLEDGEMENTS}

This research was supported by the EU-funded Hungarian grant EFOP-3.6.1-16-2016-00008.

\section{FUNDING}

Open access funding provided by University of Szeged (SZTE).

Open Access This article is distributed under the terms of the Creative Commons Attribution 4.0 International License (http://creativecommons.org/licenses/by/4.0/), which permits unrestricted use, distribution, and reproduction in any medium, provided you give appropriate credit to the original author(s) and the source, provide a link to the Creative Commons license, and indicate if changes were made.

\section{REFERENCES}

1. Pallagi E, Ambrus R, Szabó-Révész P, Csóka I. Adaptation of the quality by design concept in early pharmaceutical development of an intranasal nanosized formulation. Int J Pharm. 2015;491(1-2): 384-92.

2. Beg S, Jain S, Kushwah V, Bhatti GK, Sandhu PS, Katare O, et al. Novel surface-engineered solid lipid nanoparticles of rosuvastatin calcium for low-density lipoprotein-receptor targeting: a quality by design-driven perspective. Nanomedicine. 2017;12:333-56.

3. Csoka I, . Pallagi E, Paal TL . Extension of quality-by-design concept to the early development phase of pharmaceutical R\&D processe. Drug Discov Today 2018;23, 1340, 1343.

4. Mofrad AE, Moheb A, Masigol M, Sadeghi M, Radmanesh F. An investigation into electrochemical properties of poly (ether sulfone)/ poly (vinyl pyrrolidone) heterogeneous cation-exchange membranes by using design of experiment method. J Colloid Interface Sci. 2018 Dec;532:546-56.

5. Rahman Z, Zidan AS, Habib MJ, Khan MA. Understanding the quality of protein loaded PLGA nanoparticles variability by Plackett-Burman design. Int J Pharm. 2010;389(1-2):186-94.

6. Rakić T, Kasagić-Vujanović I, Jovanović M, Jančić-Stojanović B, Ivanović D. Comparison of full factorial design, central composite design, and box-Behnken Design in Chromatographic Method Development for the determination of fluconazole and its impurities. Anal Lett. 2014;47:1334-47.

7. Mehta A, Marso SP, Neeland IJ. Liraglutide for weight management: a critical review of the evidence. Obes Sci Pract [nternet. 2017;3(1):3-14. Available from. https://doi.org/10.1002/osp4.84.

8. Lin Y, Krogh-Andersen K, Pelletier J, Marcotte H, Östenson CG, Hammarström L. Oral delivery of pentameric glucagon-like peptide- 1 by recombinant lactobacillus in diabetic rats. PLoS One. 2016;11(9).

9. Shamekhi F, Tamjid E, Khajeh K. Development of chitosan coated calcium-alginate nanocapsules for oral delivery of liraglutide to diabetic patients. Int J Biol Macromol. 2018;120:460-7.

10. Ismail R, Csóka I. Novel strategies in the oral delivery of antidiabetic peptide drugs- insulin, GLP 1 and its analogs. Eur J Pharm Biophar. 2017;115:257-67.

11. Ismail R, Csóka I. Up to date advances in nano-carrier systems for oral delivery of antidiabetic peptides. In J Nanomed Nanotechnol. 2017:63 Available from: https://www.omicsonline.org/ conference-proceedings/2157-7439-C1-049-007.pdf.

12. Reix N, Parat A, Seyfritz E, Van Der Werf R, Epure V, Ebel N, et al. In vitro uptake evaluation in Caco-2 cells and in vivo results in diabetic rats of insulin-loaded PLGA nanoparticles. Int J Pharm. 2012;437(1-2):213-20.

13. Haggag YA, Faheem AM, Tambuwala MM, Osman MA, ElGizawy SA, O???Hagan B, et al. Effect of poly (ethylene glycol) content and formulation parameters on particulate properties and intraperitoneal delivery of insulin from PLGA nanoparticles prepared using the double-emulsion evaporation procedure. Pharm Dev Technol. 2017;1-12.

14. Park MH, Baek JS, Lee CA, Cho CW. Effect of chitosan on physicochemical properties of exenatide-loaded PLGA nanoparticles. J Pharm Investig. 2013;43(6):489-97.

15. Araújo F, Shrestha N, Gomes MJ, Herranz-Blanco B, Liu D, Hirvonen JJ, et al. In vivo dual-delivery of glucagon like peptide-1 GLP-1) and dipeptidyl peptidase-4 (DPP4) inhibitor through composites prepared by microfluidics for diabetes therapy. Nanoscale. 2016;8(20):10706-13.

16. Cohen-Sela E, Chorny M, Koroukhov N, Danenberg HD, Golomb G. A new double emulsion solvent diffusion technique 
for encapsulating hydrophilic molecules in PLGA nanoparticles. J Control Release. 2009;133(2):90-5.

17. Tefas LR, Tomuță I, Achim M, Vlase L. Development and optimization of quercetin-loaded PLGA nanoparticles by experimental design. Clujul Med. 2015;88(2):214.

18. Ismail R, Csóka I. Quality by design (QbD) oriented study for designing new oral Liraglutide loaded polymeric nanoparticles. In: 11th world meeting on pharmaceutics, biopharmaceutics and pharmaceutical technology, Granada, Spain. 2018. p. 50.

19. Ismail R, Csóka I. Evaluation of techniques and possibilities for oral delivery of antidiabetic peptide drug - liraglutide - in a novel delivery system. In: Acta pharmaceutica Hungarica. 2017. p. 132-3.

20. Pallagi E, Ismail R, Paal TL, Csoka I. Initial risk assessment as part of the quality by design in peptide drug containing formulation development. Eur J Pharm Sci. 2018 Sep;122:160-9.

21. Fernández EF, Santos-Carballal B, de Santi C, Ramsey JM, MacLoughlin R, Cryan SA, et al. Biopolymer-based nanoparticles for cystic fibrosis lung gene therapy studies. Materials (Basel). 2018;11(1).

22. Poddar A, Sawant KK. Optimization of Galantamine loaded bovine serum albumin nanoparticles by quality by design and its preliminary characterizations. J Nanomed Nanotechnol [Internet]. 2017;8(5):459. Available from:. https://doi.org/10.4172/2157$7439.1000459 \% 0 \mathrm{~A}$

23. Akl MA, Kartal-Hodzic A, Oksanen T, Ismael HR, Afouna MM, Yliperttula M, et al. Factorial design formulation optimization and in vitro characterization of curcumin-loaded PLGA nanoparticles for colon delivery. J Drug Deliv Sci Technol. 2016;32:10-20.

24. Taghipour B, Yakhchali M, Haririan I, Tamaddon AM, Samani SM. The effects of technical and compositional variables on the size and release profile of bovine serum albumin from PLGA based particulate systems. Res Pharm Sci. 2014;9(6):407-20.

25. Mainardes RM, Evangelista RC. PLGA nanoparticles containing praziquantel: effect of formulation variables on size distribution. Int J Pharm. 2005;290(1-2):137-44.

26. Zhang L, He Y, Ma G, Song C, Sun H. Paclitaxel-loaded polymeric micelles based on poly( $\varepsilon$-caprolactone)-poly (ethylene glycol)poly( $\varepsilon$-caprolactone) triblock copolymers: in vitro and in vivo evaluation. Nanomed Nanotechnol. Biol Med. 2012;8(6):925-34.

27. Dewangan HK, Pandey T, Maurya L. S. S. Rational design and evaluation of $\mathrm{HBsAg}$ polymeric nanoparticles as antigen delivery carriers. Int J Biol Macromol. 2018;111:804-12.

28. Abdelkader DH, El-Gizawy SA, Faheem AM, McCarron PA, Osman MA. Effect of process variables on formulation, in-vitro characterisation and subcutaneous delivery of insulin PLGA nanoparticles: an optimisation study.J Drug Deliv Sci Technol. 2018;43: 160-71.

29. Haggag Y, Abdel-Wahab Y, Ojo O, Osman M, El-Gizawy S, ElTanani M, et al. Preparation and in vivo evaluation of insulinloaded biodegradable nanoparticles prepared from diblock copolymers of PLGA and PEG. Int J Pharm. 2016;499(1-2):236-46.

30. Fonte P, Soares S, Costa A, Andrade JC, Seabra V, Reis S, et al. Effect of cryoprotectants on the porosity and stability of insulinloaded PLGA nanoparticles after freeze-drying. Biomatter. 2012;2(4):329-39

31. Halayqa M, Domańska U. PLGA biodegradable nanoparticles containing perphenazine or chlorpromazine hydrochloride: effect of formulation and release. Int J Mol Sci. 2014;15(12):23909-23.
32. Francisca A, Shrestha N, Gomes MJ, Herranz-Blanco B, Liu D, Hirvonen J, et al. In vivo dual-delivery of glucagon like peptide -1 (GLP-1) and dipeptidyl peptidase-4 (DPP4) inhibitor through composites prepared by microfluidics for diabetes therapy. Nanoscale , 2016,8, 10706-10713.

33. Rescignano N, Fortunati E, Armentano I, Hernandez R, Mijangos $\mathrm{C}$, Pasquino R, et al. Use of alginate, chitosan and cellulose nanocrystals as emulsion stabilizers in the synthesis of biodegradable polymeric nanoparticles. J Colloid Interface Sci. 2015 May;445:31-9.

34. Feczkó T, Tóth J, Dósa G, Gyenis J. Optimization of protein encapsulation in PLGA nanoparticles. Chem Eng Process Process Intensif. 2011:50:757-65.

35. Rahman Z, Zidan AS, Habib MJ, Khan MA. Understanding the quality of protein loaded PLGA nanoparticles variability by Plackett-Burman design. Int J Pharm. 2010;389:186-94.

36. Jain A, Jain SK. Formulation and optimization of temozolomide nanoparticles by 3 factor 2 level factorial design. Biomatter. 2013;3(2).

37. Narayanan K, Subrahmanyam VM, Venkata Rao J. A fractional factorial design to study the effect of process variables on the preparation of hyaluronidase loaded PLGA nanoparticles. Enzyme Res. 2014;2014:1-10.

38. Almalik A, Alradwan I, Kalam MA, Alshamsan A. Effect of cryoprotection on particle size stability and preservation of chitosan nanoparticles with and without hyaluronate or alginate coating. Saudi Pharm J. 2017;25(6):861-7.

39. Mali N, Wavikar P, Vavia P. Serratiopeptidase loaded chitosan nanoparticles by polyelectrolyte complexation: in vitro and in vivo evaluation. AAPS PharmSciTech. 2015 Feb;16(1):59-66.

40. Fonte P, Soares S, Sousa F, Costa A, Seabra V, Reis S, et al. Stability study perspective of the effect of freeze-drying using cryoprotectants on the structure of insulin loaded into PLGA nanoparticles. Biomacromolecules. 2014;15(10):3753-65.

41. Abdelwahed W, Degobert G, Stainmesse S, Fessi H. Freeze-drying of nanoparticles: formulation, process and storage considerations. Adv Drug Deliv Rev. 2006;58:1688-713.

42. Ding Y, ZhengJ, Xia X, Ren T, KanJ. Box-Behnken design for the optimization of nanoscale retrograded starch formation by highpower ultrasonication. LWT Food Sci Technol. 2016;67:206-13.

43. Wang Y, Li P, Kong L, Peng Z, Luo Y. Formulation optimization for high drug loading colonic drug delivery carrier. IEEE. 2010: 1686-9.

44. Fonte P, Andrade F, Azevedo C, Pinto J, Seabra V, van de Weert $\mathrm{M}$, et al. Effect of the freezing step in the stability and bioactivity of protein-loaded PLGA nanoparticles upon Lyophilization. Pharm Res. 2016;33(11):2777-93.

45. Park MH, Jun HS, Jeon JW, Park JK, Lee BJ, Suh GH, et al. Preparation and characterization of bee venom-loaded PLGA particles for sustained release. Pharm Dev Technol. 2016:1-8.

Publisher's Note Springer Nature remains neutral with regard to jurisdictional claims in published maps and institutional affiliations. 